\title{
Integration Modelica with Digital Mockup Tool using the FMI
}

\author{
Shinji Matsuda $^{1}$ Hiroshi Toriya ${ }^{1}$ Hiromasa Suzuki $^{2} \quad$ Koichi Ohtomi $^{2}$ \\ ${ }^{1}$ Lattice Technology Co.,Ltd., Japan, \{matsuda, toriya elattice.co.jp \\ ${ }^{2}$ Department of Precision Engg., The University of Tokyo, Japan, suzuki@den.t.u-tokyo.ac.jp \\ koichi.ohtomi@delight.t.u-tokyo.ac.jp
}

\begin{abstract}
The Delight Design Platform Project is managed by The University of Tokyo as part of the Crossministerial Strategic Innovation Program (SIP) which is organized by Cabinet Office, Government of Japan. This project recommends using 1DCAE design tools in the concept phase of the product development. In the Delight Design Platform, new product concepts are simulated and evaluated as 1DCAE models. One of the objectives of this project is to prototype a tool for translating product concepts to 3D models. This paper describes a method of integrating Modelica with a 3D digital mockup (DMU) tool. The prototype is implemented as an extension of XVL Studio, which is a popular DMU tool provided by Lattice Technology Co.,Ltd. The integration is implemented using the FMI (Functional Mockup Interface).
\end{abstract}

Keywords: $\quad$ Modelica, 1DCAE, Functional Mockup Interface, XVL, Digital Mockup

\section{Introduction}

For many years, Japanese manufacturers placed top priority on developing high quality products at low cost. However, nowadays their primary focus is to develop more attractive products (Ohtomi K, 2015). When thinking about attractive product design, we focused on the fact that there are many potentially interesting product ideas left unattended in the backyard of the companies without being commercialized. In the Delight Design Platform Project, we are trying to support the development of attractive products by visualizing the attractive qualities of new ideas. The technology of Model Based Design (MBD), represented by Modelica, is one of the key technologies in our project. In this paper, we propose a method to visualize the MBD simulation results in a DMU tool using FMI. By integrating with the DMU tool, the simulation results can be represented with realistic 3D graphics which makes the presentation more impressive and persuasive. Also, the DMU tool has another useful feature - it supports the inclusion of a 3D human model, which is a very effective way to perform human work analyses. For example, product usability can be evaluated using estimates for product characteristics such as weight and size, but with an MBD human model it is possible to directly evaluate the load on the human body. In our prototype, we tried to perform a realistic evaluation by modeling the product along with a human model.

This paper describes the integration of Modelica and DMU tools and some of the resulting outputs.

Figure 1 shows the outline of the integration.

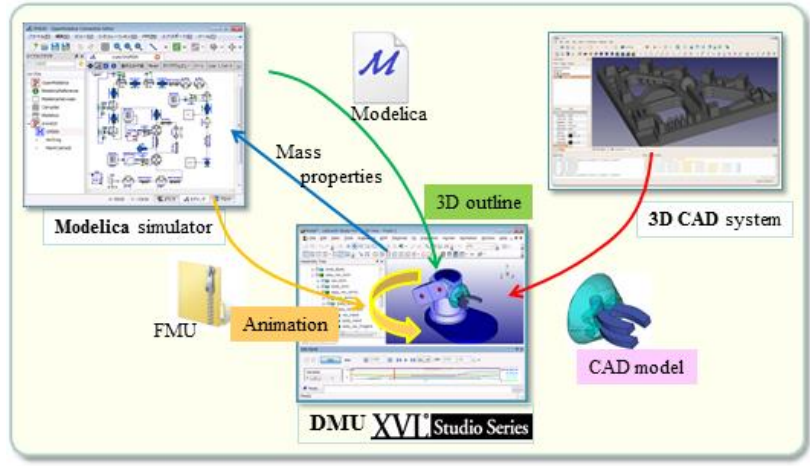

Figure 1 Outline of the integration Modelica with $X V L$ Studio.

\section{XVL and DMU Tool}

XVL® (eXtensible Virtual reality description Language) is a lightweight 3D file format developed by Lattice Technology Co.,Ltd. And XVL Studio is a digital mockup tool based on the XVL technology (Lattice Technology, 2016). It is widely used for design review, digital assembly and generating technical illustrations (Toriya H. 2008).

The file size of a complete model of an automobile, a piece of construction equipment, an agricultural machine, a train or a ship can easily reach several GB, and it is difficult for CAD systems to handle such large assemblies. In these cases a lightweight 3D file format such as $X V L$ is more effective (Toriya H. 2014; Toriya H., Jablonski M., 2017).

Digital Assembly is the concept of performing a virtual assembly validation without using an actual prototype. It requires managing multiple structures for each product. Using $X V L$ it is possible to define multiple structures in a single file -- for design, for manufacturing and for service. This makes it possible 
for the user to easily change the design structure to the desired structure, such as the manufacturing structure.

$\mathrm{XVL}$ is also capable of handling the large point cloud data generated by laser scanners. 3D point cloud data is a new technology that makes it easy to convert existing facilities to 3D models. For example, a 3D point cloud scan of a facility makes it possible to check whether new equipment will fit in the existing space. The following chapters will include an example of using 3D point cloud data.

\section{3D Model Generation}

In the libraries provided by Modelica we want to highlight here is MultiBody library. Since MultiBody library is targeting to 3D mechanical system, we integrate the library with a DMU tool for visualizing mechanical simulation.

\subsection{Geometry}

Some of the Commercial products for Modelica modeling support 3D visualization (Figure 2). But it is limited only for visualization purpose. In most cases, reuse of the visualization data is not considered.

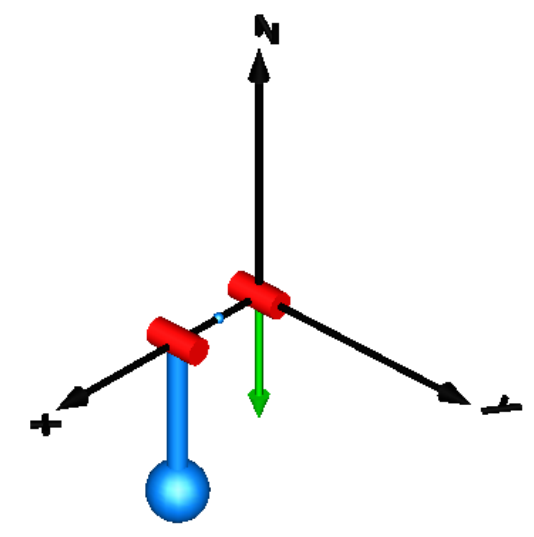

Figure 2 3D visualization in Dymola (Dassault Systèmes, 2016).

Each component in the Multibody library has the parameters of the visualization such as the sphere diameter or the length of the cylinder. We have implemented the parser, which parses the information of the visualization, and a generator which generates B-Spline surface using XVL Kernel. Most of the Modelica tools support only polygon data as 3D model. However, NURBS models are more common than polygon models in 3D CAD system (Figure 3 ). The 3D model can be exported as IGES file format with the standard command of XVL Studio. The IGES files can be imported to most of the CAD systems.

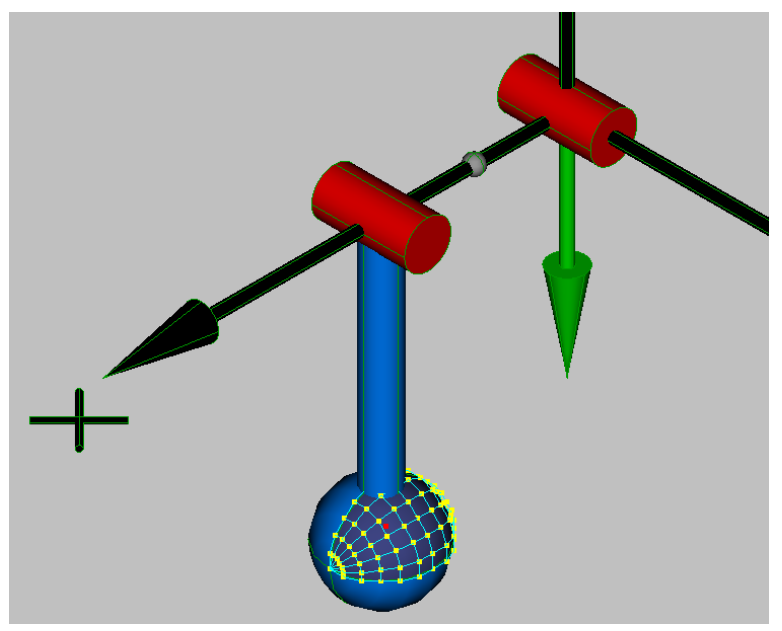

Figure 3 3D model (with NURBS surface)

Our prototype generates 3D models from the Modelica components listed in the Table 1.

Table 1 Components supported in the prototype.

\begin{tabular}{|l|l|}
\hline Package & Model \\
\hline MultiBody & World \\
\hline MultiBody.Joints & Prismatic \\
\hline MultiBody.Joints & Revolute \\
\hline MultiBody.Joints & Cylindrical \\
\hline MultiBody.Joints & Universal \\
\hline MultiBody.Joints & Spherical \\
\hline MultiBody.Joints & SphericalSpherical \\
\hline MultiBody.Joints & UniversalSpherical \\
\hline MultiBody.Joints & JointUPS \\
\hline MultiBody.Parts & Fixed \\
\hline MultiBody.Parts & FixedTranslation \\
\hline MultiBody.Parts & Body \\
\hline MultiBody.Parts & BodyShape \\
\hline MultiBody.Parts & BodyBox \\
\hline MultiBody.Parts & BodyCylinder \\
\hline MultiBody.Parts & PointMass \\
\hline
\end{tabular}

\subsection{Assembly Structure}

The CAD system or the DMU tool has an assembly structure which is defined as a tree structure (Figure 4).

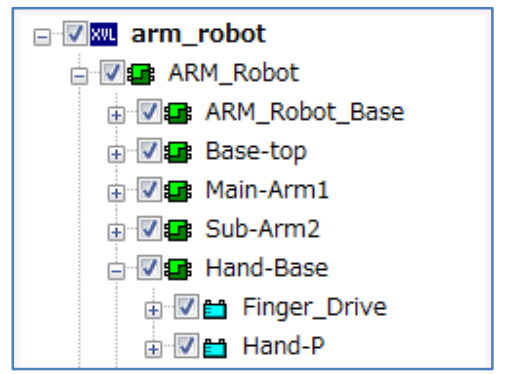

Figure 4 An assembly tree in the XVL Studio.

The assembly structure has an important functionality in CAD system (also in DMU Tool), that is locating its sub-components in the model coordinate system. An assembly has the information of its sub- 
components and the transformation matrix for each sub-component. User can move a sub-component by changing its translation matrix. This functionality can be used to animating the simulation result in the DMU Tool. The appropriate structure should be created while generating the 3D model from Modelica. For example a Body component connected to a Revolute joint, has to be moved synchronizing with the rotation of the joint (Figure 5). To do this, the 3D model of the joint and the model of Body should belong to one assembly group (Figure 6).

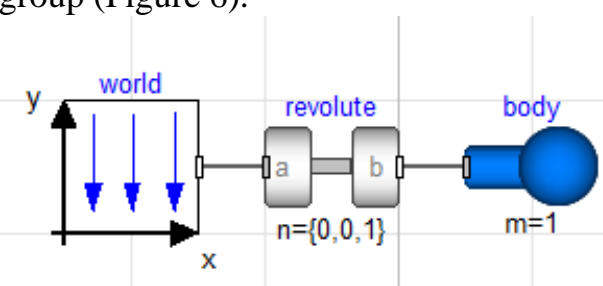

Figure 5 Example model with a Revolute and a Body.

$$
\begin{aligned}
& \checkmark \text { 『 example } \\
& \text { †回自 world } \\
& \text { 由、四凹 body } \\
& \text { †目国 revolute } \\
& \text { 由回自 prt_body }
\end{aligned}
$$

Figure 6 Assembly tree of the example, which shows the shape of the body "prt_body" and the shape of revolute "revolute" are included in one assembly "body".

\section{Kinematics}

The MultiBody Library of Modelica is designed to simulate both kinematic and dynamic mechanical models (Martin Otter, et al. 2003). The analysis of kinematics is one of the most popular features of 3D CAD systems. XVL Studio (A DMU Tool) also has the functionality of the kinematic analysis. The revolute joint, the linear sliding mechanism and some other types of kinematic objects can be defined in $X V L$ Studio (Figure 7).

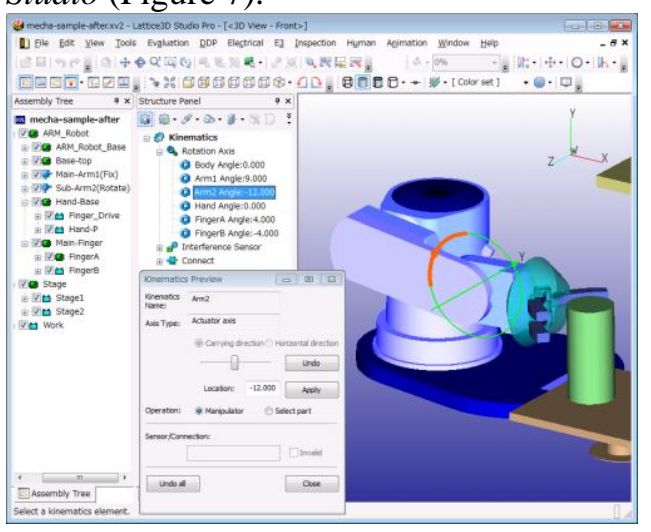

Figure 7 GUI of the kinematic analysis in XVL Studio.
While reading the Modelica model, the kinematic information is translated to the kinematic objects of XVL Studio. For example the information of the revolute joint of the Modelica is translated to the information of the Rotation Axis object in XVL Studio. It has following information as the kinematic object (Table 2).

Table 2 The kinematic information of the Rotation Axis in XVL Studio.

\begin{tabular}{|l|l|}
\hline information & data type \\
\hline Joint Name & text \\
\hline Point of the origin & vector \\
\hline Rotational Axis & vector \\
\hline Rotational part & text \\
\hline Fixed part & text \\
\hline \multicolumn{1}{|l|}{ An instance of the revolute joint defined as }
\end{tabular}

"revolute1" in a Modelica file is translated as follows.

The instance name of the Modelica is translated to the name of the Rotation Axis of XVL. The Point of the Origin of XVL is calculated by traversing the connection of the Modelica model. The parameter " $n$ " of the revolute joint is translated to the vector of the rotational axis in XVL. The Rotational part and the fixed part in the XVL are also found by traversing the connection of the Modelica model.

This information is used for the $3 \mathrm{D}$ animation in XVL Studio.

\section{Running Simulation}

The DMU Tool does not have the functionality to generate the executable module for simulation. The prototype runs the simulation using FMI. We used FMI Library by JModelica.org in our prototype for running the simulation. FMU module is generated by using a small batch command. Following is an example batch command for JModelica.

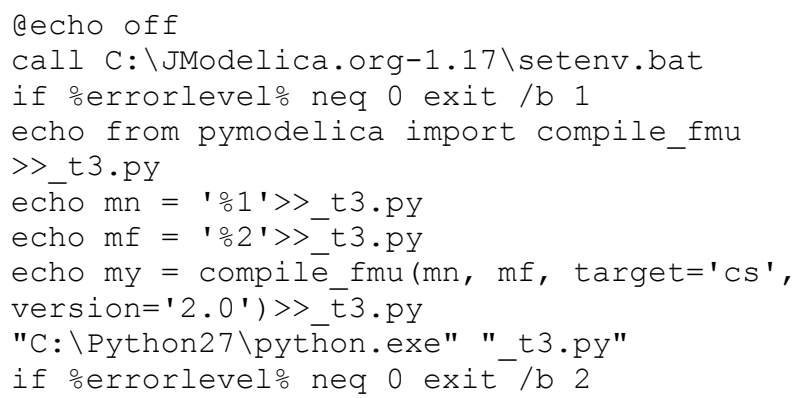

This batch file takes 2 arguments. One is the name of the model, and the other is the name of the Modelica file. It generates a python script as _t3.py and call python.exe.

\subsection{FMI Interface}

The FMI (Functional Mockup Interface) is the standard which enables running the simulation from any tools (FMI-Standard.org, 2014). There are publicly available 
FMI implementations. For example, FMI Library (JModelica.org, 2016) is a library developed by JModelica.org can be downloaded in source code or binaries for Windows. Using the library, all of the steps for running the simulation can be executed by simple function call, like unzipping the FMU module or parsing the modelDescription.xml. Our prototype is implemented to use the FMI Library.

\subsection{D Matrix}

The connector of the MultiBody Library is defined as the Frame. Frame model is defined in the package Interfaces as following.

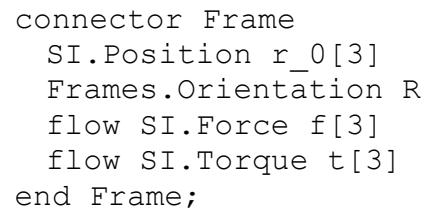

The position vector $r_{-} 0$ is directed from the origin of the world coordinate system to the origin of the Frame. The orientation object $\mathrm{R}$ describes the relative orientation between the world frame and the Frame. From these parameters, we can generate a transformation matrix which is used to animate the 3D object in DMU Tool.

$$
|A|=\left|\begin{array}{cccc}
R \cdot T[1,1] & R \cdot T[2,1] & R \cdot T[3,1] & r_{-} 0[1] \\
R \cdot T[1,2] & R \cdot T[2,2] & R \cdot T[3,2] & r_{-} 0[2] \\
R \cdot T[1,3] & R \cdot T[2,3] & R \cdot T[3,3] & r_{-} 0[3] \\
0 & 0 & 0 & 1
\end{array}\right|
$$

This translation matrix moves the 3D object in the DMU model. The values r_0 and R.T of the components in the Modelica model are referred while running the simulation and are used for the animation of 3D model.

\subsection{Using CAD Model}

Using the 3D CAD model of the existing products, the presentation of the simulation result becomes more realistic. The DMU Tool has the functionality to import CAD model. Most of the DMU Tools support many types of the CAD format. Figure 8 is the list of the supported formats in XVL Studio.

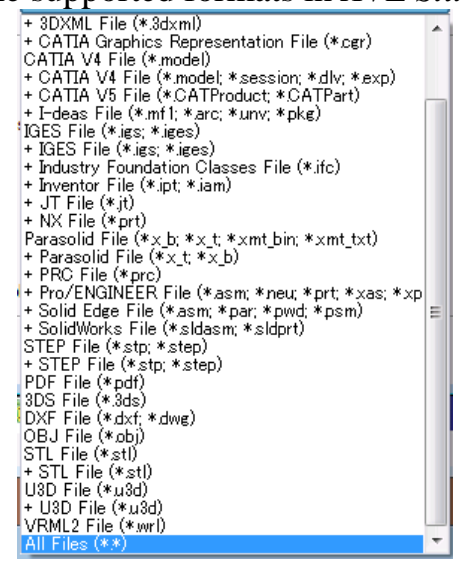

Figure 8 CAD format supported by XVL Studio.
To use the existing CAD data in our prototype, just drag and drop the CAD file to XVL Studio. Following instructions show how to use the CAD data in the prototype.

- Import Modelica model to the prototype.

- The 3D visualization model is generated. (Figure 9)

- Import CAD data to DMU Tool. (Figure 10)

- Move parts of the CAD model to the group under the visualization model. (Figure 11)

- Run simulation. (Figure 12)

Using the integration Modelica and DMU Tool the simulation can be visualized with CAD model with simple operations like this.

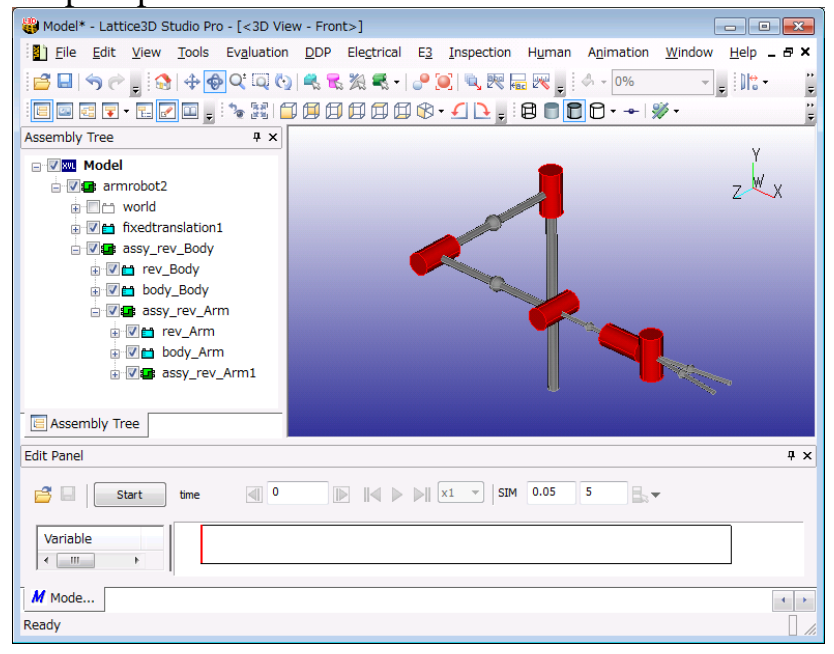

Figure 93 D visualization model generated with the prototype.

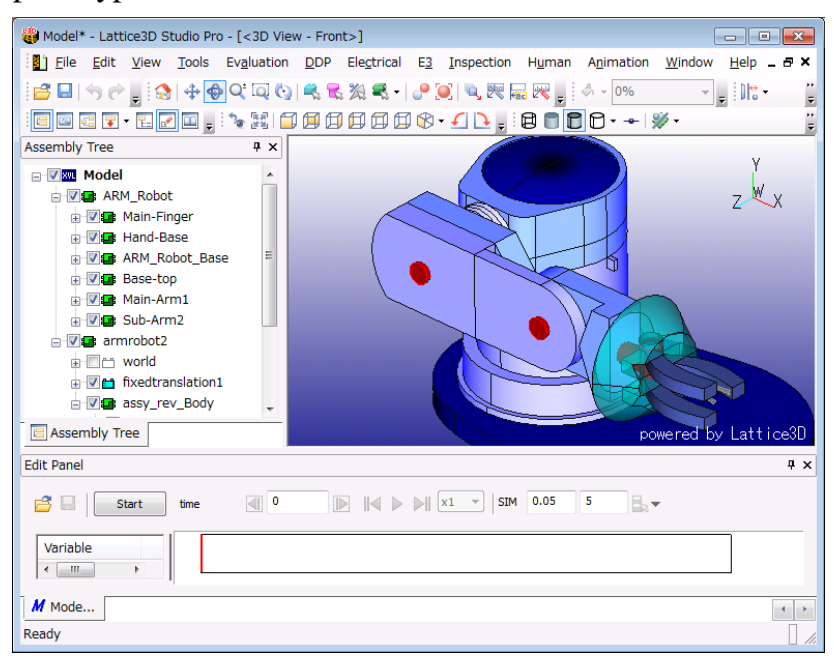

Figure $10 \mathrm{CAD}$ model is imported over the visualization model. 


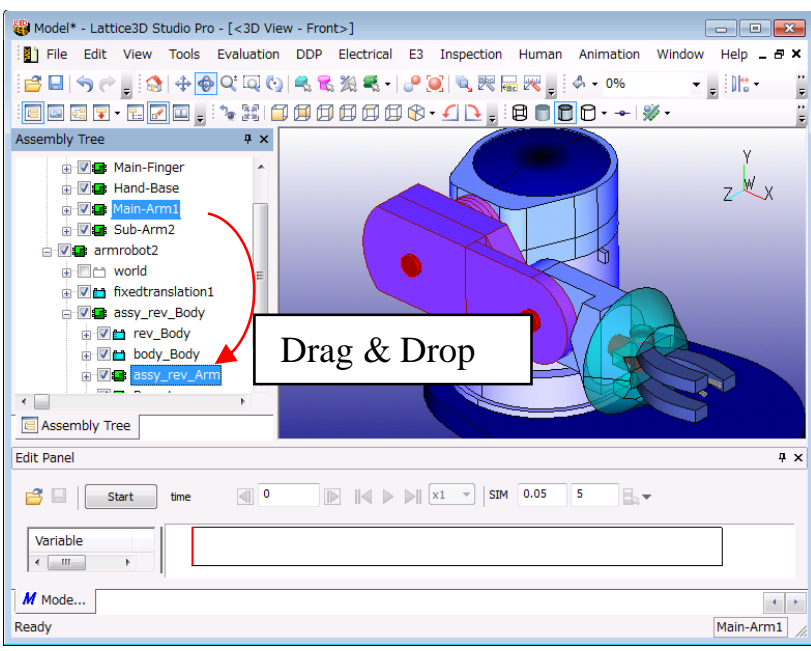

Figure 11 Move CAD model to the group generated with the $3 \mathrm{D}$ visualization model.

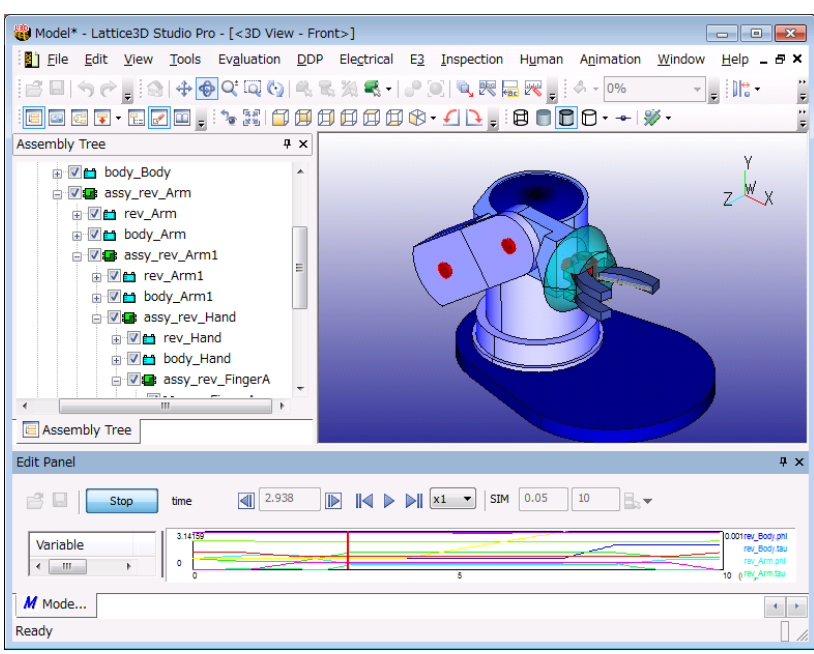

Figure 12 Simulation is visualized with the CAD model.

\section{Example}

We create a crane model in our project. The CAD data of a crane is provided by a Japanese manufacturer of mobile cranes.

\subsection{Crane Model}

Figure 13 is a crane model created with Dymola. It consists of 2 revolute joints for simulating the fall over problem. 2 revolute joints and 4 prismatic joints are for simulating the movement of the boom. One prismatic joint held in the revolute joint and a universal joint are for the extending of the wire. Figure 14 is a screenshot of the prototype showing the 3D model generated from the Modelica file. As instructed in chapter 5.3, CAD model of the product can be imported (Figure 15). Figure 16 is the screenshot of the final 3D model, which includes the 3D skeleton model generated from the visualization information defined in Modelica, $\mathrm{CAD}$ data of the product design and point cloud of the construction field. The point cloud is measured with the laser scanner and imported to XVL Studio.

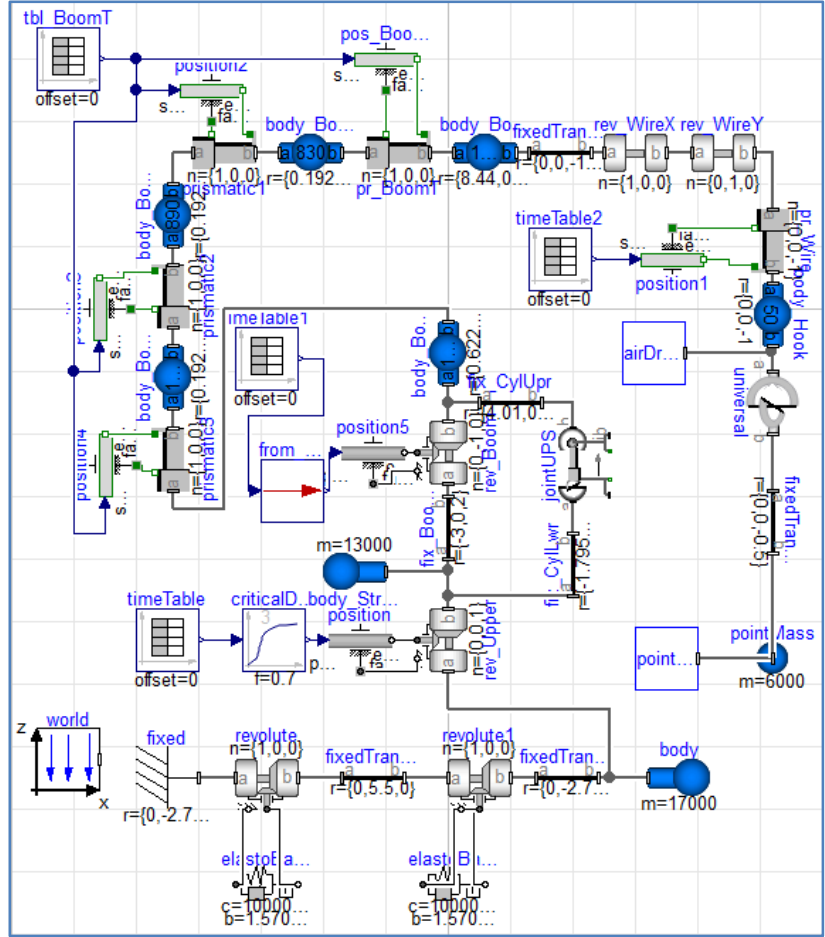

Figure 13 Modelica model of a mobile crane.

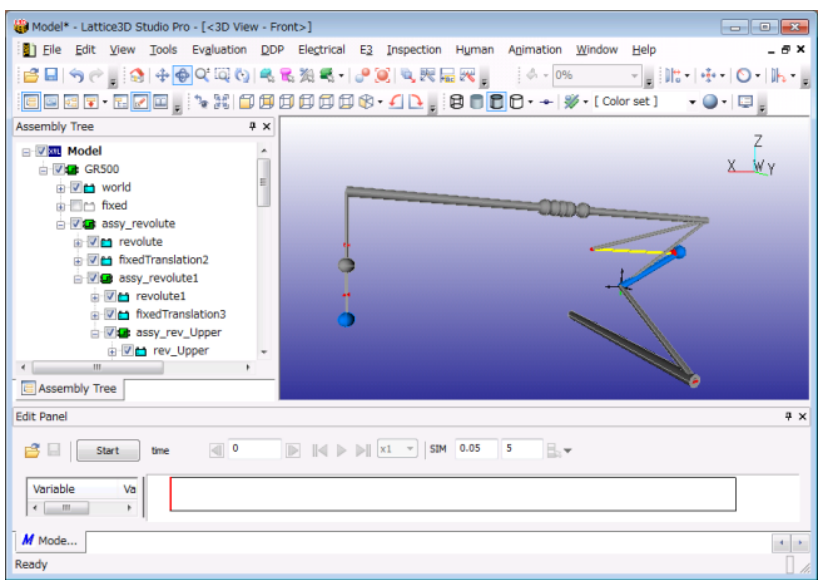

Figure $143 \mathrm{D}$ skeleton data generated by the prototype

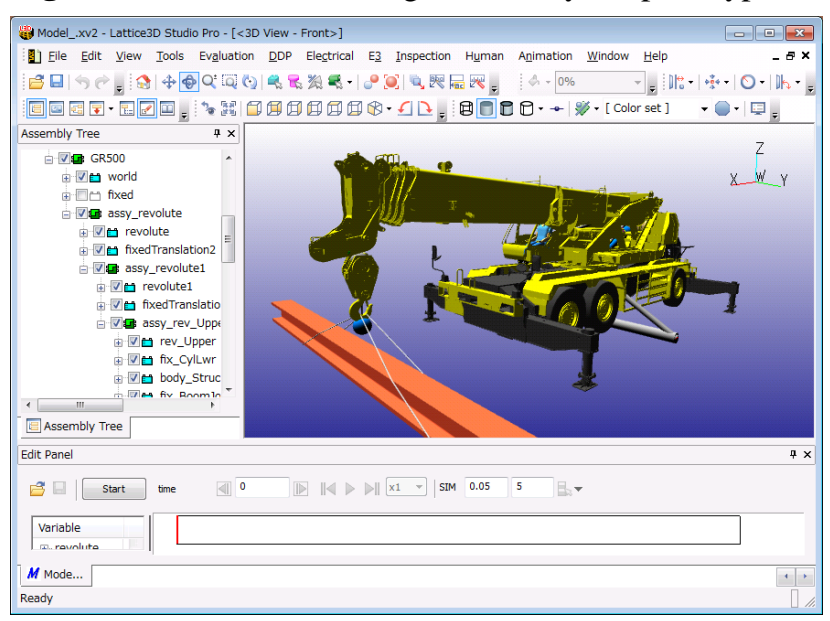

Figure 15 A screen shot after imported the CAD model. 


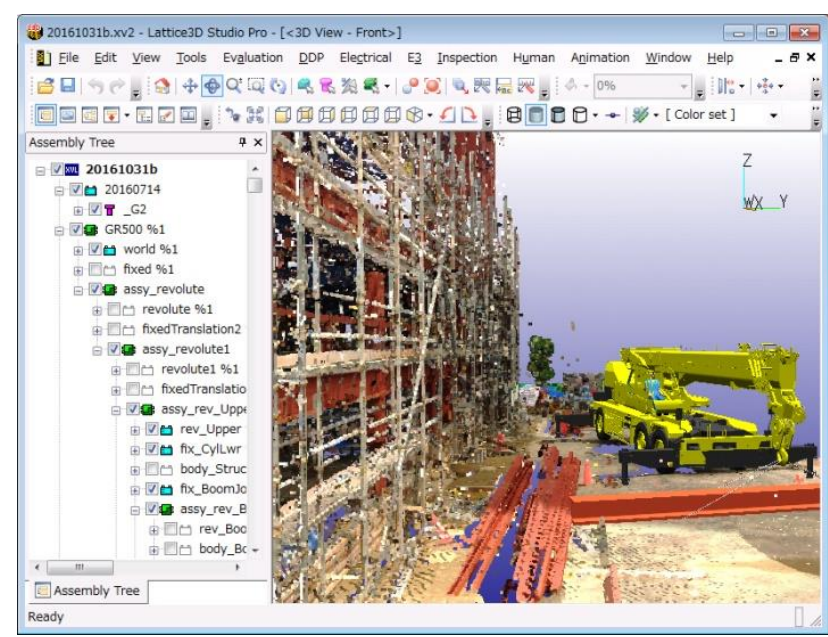

Figure 16 A screen shot after imported point cloud.

Using this model, we have simulated the swing of the load while turning the Boom (Figure 17). Figure 18 shows the simulation of the problem of fall over of the full vehicle.

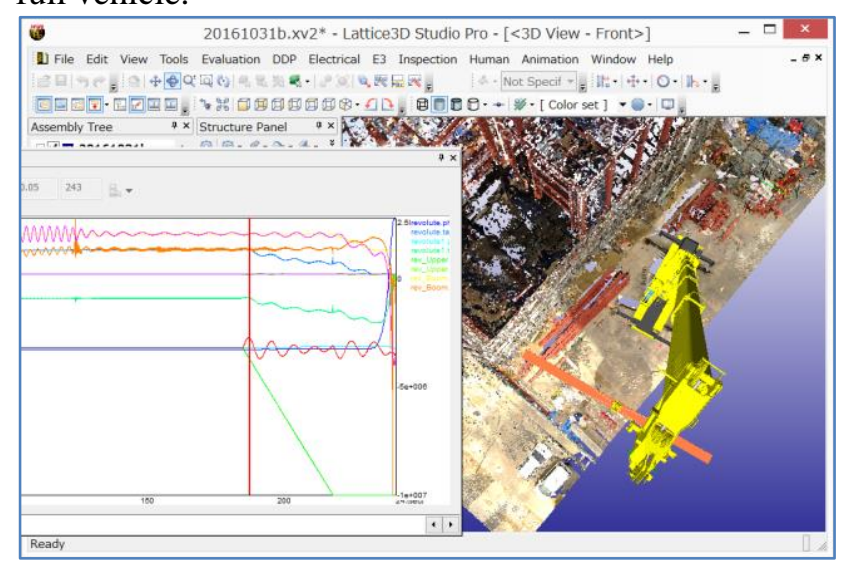

Figure 17 A screenshot simulating the swing of the load while turning the Boom.

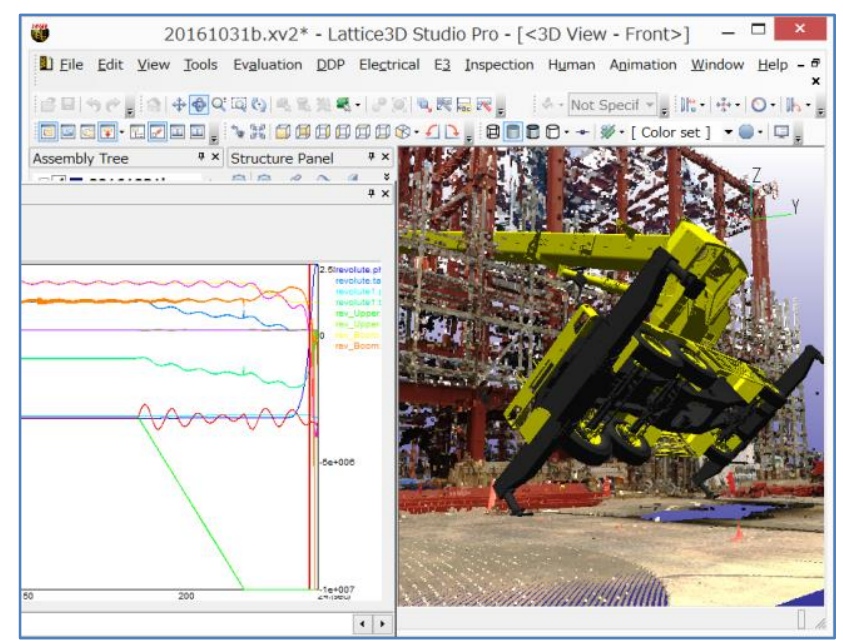

Figure 18 Simulating the problem of fall over when the boom was tilted.

\section{Human Model}

Analyzing the human interaction, during the production in the factory is one of the important usecase of the DMU tool. Some of the DMU Tools has the functionality of evaluate the posture of the worker using 3D human model. On the other hand there are some biomechanics software enables to analyze the human muscle bone model. For example DhaibaWorks developed by National Institute of Advanced Industrial Science and Technology (AIST, 2016) is well known in Japan. These bio-mechanics software are for the expert users in the laboratory of the universities or the enterprise. Our target in the Delight Design Platform is to develop a human model easy to use for engineers in the manufacturer using the Modelica technology.

\subsection{Muscle-Bone Model Prototype}

Our first prototype was a generator, which generate a simple 3D muscle-bone model using the kinematics of $X V L$. The generator reads a BVH file, and generates a $X V L$ model referring the structure data in the BVH file. The BVH file format is originally developed by Biovision as a motion capture data file format (Autodesk, 2016).

In this model, there are 16 skeletal joints. We defined degree-of-freedom for each joint, and associated prime mover muscle (Table 3 ).

Table 3 Mapping of the skeletal joint and muscle.

\begin{tabular}{|c|c|c|}
\hline skeltal joint & axis & associated muscle \\
\hline \multirow[t]{3}{*}{ Left/Right Thigh } & $\mathrm{x}$ & psoas $1 / r$ \\
\hline & $\mathrm{y}$ & gluteus I/r \\
\hline & $z$ & piriformis $I / r$ \\
\hline Left/Right Leg & $x$ & femoris $I / r$ \\
\hline \multirow[t]{2}{*}{ Left/Right Foot } & $x$ & surae $I / r$ \\
\hline & $\mathrm{y}$ & peroneus I/r \\
\hline \multirow[t]{3}{*}{ Chest } & $x$ & abdominis \\
\hline & $\mathrm{y}$ & ex oblique \\
\hline & $z$ & in oblique \\
\hline \multirow[t]{2}{*}{ Left/Right Shoulder } & $y$ & trapezius $I / r$ \\
\hline & $z$ & pectoralis $\min \mathrm{I} / \mathrm{r}$ \\
\hline \multirow[t]{3}{*}{ Left/Right Arm } & $x$ & pectoralis maj $\mathrm{I} / \mathrm{r}$ \\
\hline & $\mathrm{y}$ & deltoid I/r \\
\hline & $z$ & spinatus $I / r$ \\
\hline \multirow[t]{2}{*}{ Left/Right Forearm } & $x$ & biceps I/r \\
\hline & y & pronator $\mathrm{l} / \mathrm{r}$ \\
\hline \multirow[t]{2}{*}{ Left/Right Hand } & $x$ & digitorum I/r \\
\hline & $\mathrm{y}$ & carpi l/r \\
\hline \multirow[t]{2}{*}{ Head } & $x$ & sternocleido \\
\hline & $\mathrm{y}$ & splenius \\
\hline
\end{tabular}

The basic model of a set of joint and muscle is shown in Figure 19. 


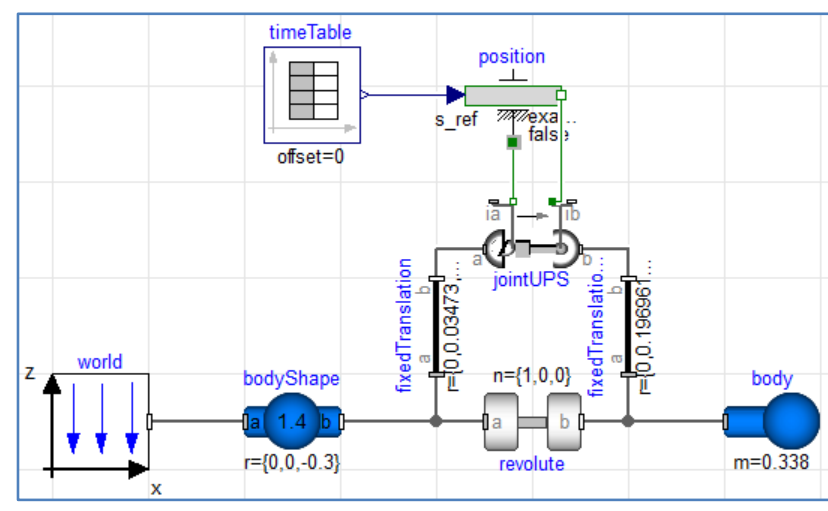

Figure 19 The basic model with one rotational joint and one JointUPS.

The Revolute component is associated to one degree of freedom of a skeletal joint. The JointUPS component is associated to the prime mover muscle. Similarly, we generated instance for all joints (Figure 20).

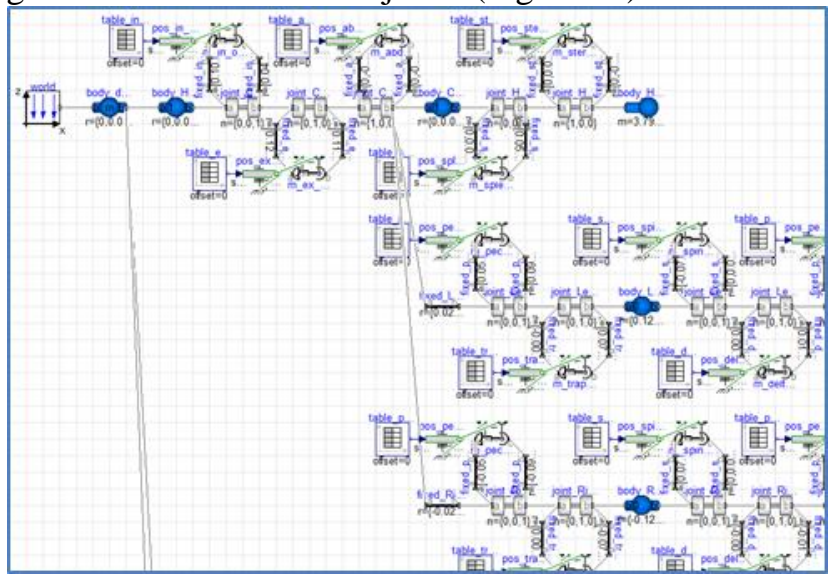

Figure 20 A Modelica model of the muscle bone model.

We prepared a 3D model with kinematic definitions referring a 3D human model for anatomy (Figure 21). The prototype generates the Modelica model from the geometric and kinematic information in the $3 \mathrm{~d}$ model.

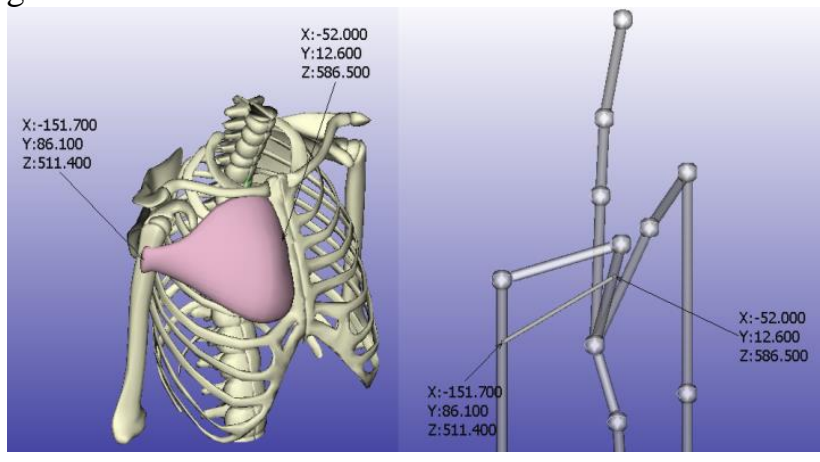

Figure 21 Defining the fixing position of the muscle to the bone. The left is an anatomy model and the right is a kinematic model.

A skin model created with CG software is added just like the CAD model written in the section 5.3 (Figure 22).

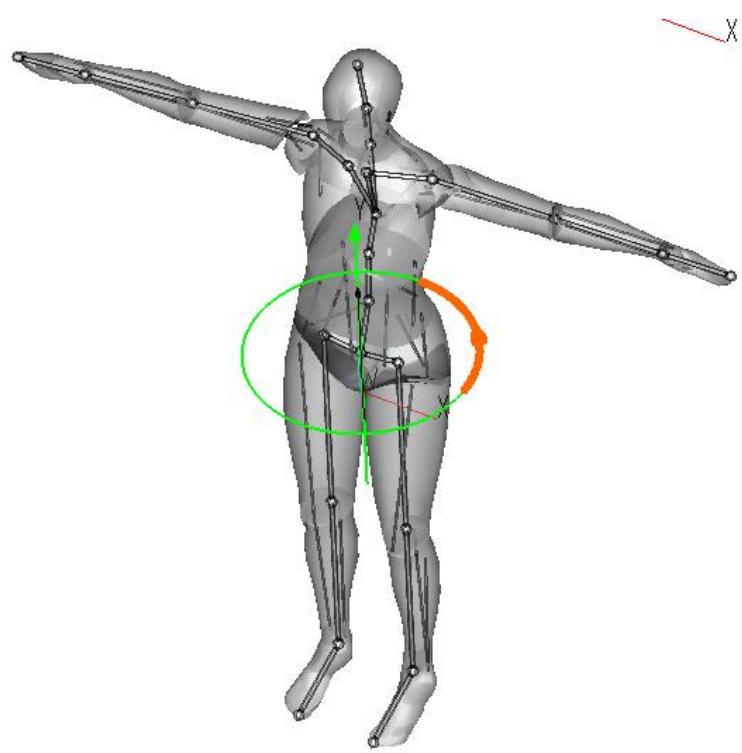

Figure 22 A 3D muscle bone model with skin.

In this model the properties of mass and the inertia tensor are calculated by the skin data of the 3D model. This concept is as same as the one in the paper Redundancies in Multibody System and Automatic Coupling of CATIA and Modelica (Hilding Elmqvist et al., 2009). Also the value in the TimeTable which is the input of the JointUPS is embedded from the value in the BVH motion data. Figure 23 is a table plot of the motion data in Dymola. The input of the JointUPS is the relative distance and it is calculated from the Euler angles of the joint contained in the BVH file.

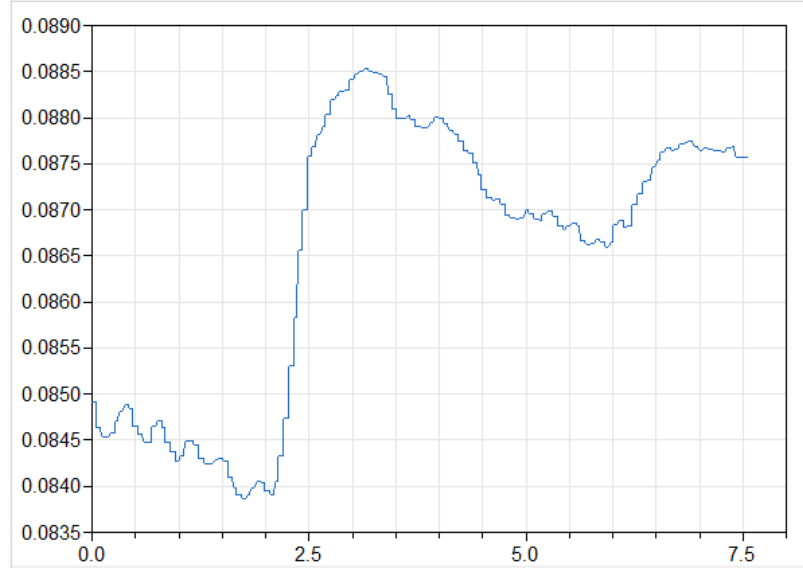

Figure 23 A table plot of the motion data embedded in the Modelica model.

We have visualized the force of the JointUPS as color mapping. Figure 24 is a sample showing the color mapping of the human model while using the hair-dryer. Our prototype generates the color mapping as a key frame animation of XVL Studio. 


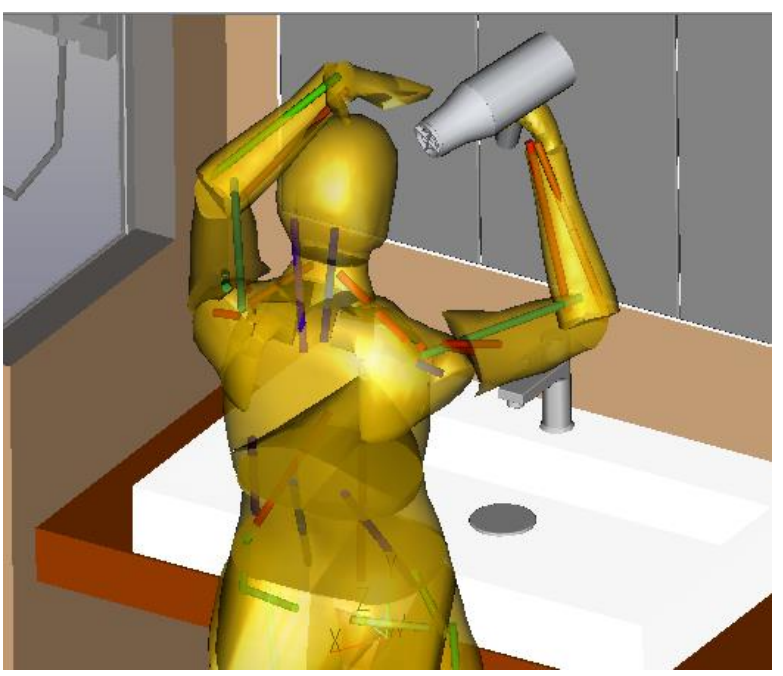

Figure 24 The visualization with the color mapping.

Following charts are samples of the simulation result. Figure 25 is a chart showing the length of the JointUPS placed at the position of the biceps. Figure 26 is the force of the JointUPS. Since the sampling rate of the motion capture is not high, the force of the JointUPS is filtered with Blocks.Continuous.Filter component.

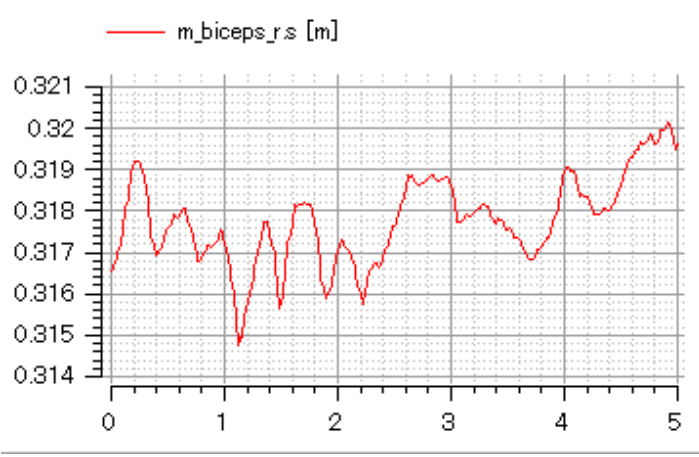

Figure 25 The length of the biceps.

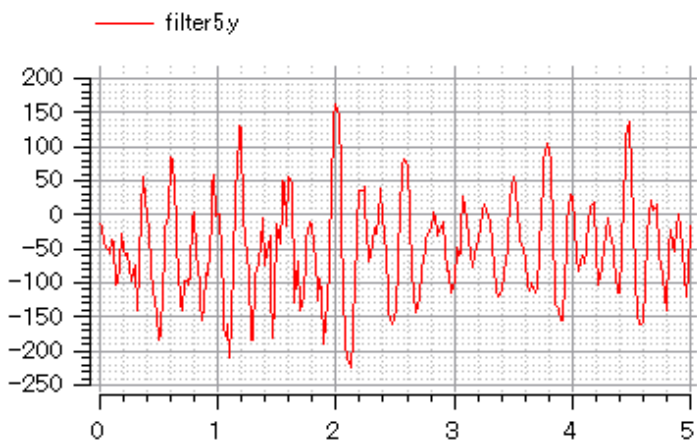

Figure 26 The force of the biceps.

\subsection{Reaction Force on the Ground}

Our first prototype described in the previous section has some problems. For example it does not simulate the reaction force from the ground. There is a contact library for the MultiBody Library, proposed as the IdealizedContact (Oestersötebier et al., 2014). Also a simple point contact model is proposed in the paper
Kinematic and Dynamic Analysis for Biped Robots Design (David M., 2012).

We have used a modified one point contact model in the following example. This model is a passive dynamic walking model, which is intended to simulate the human gait (Figure 27, Figure 28).

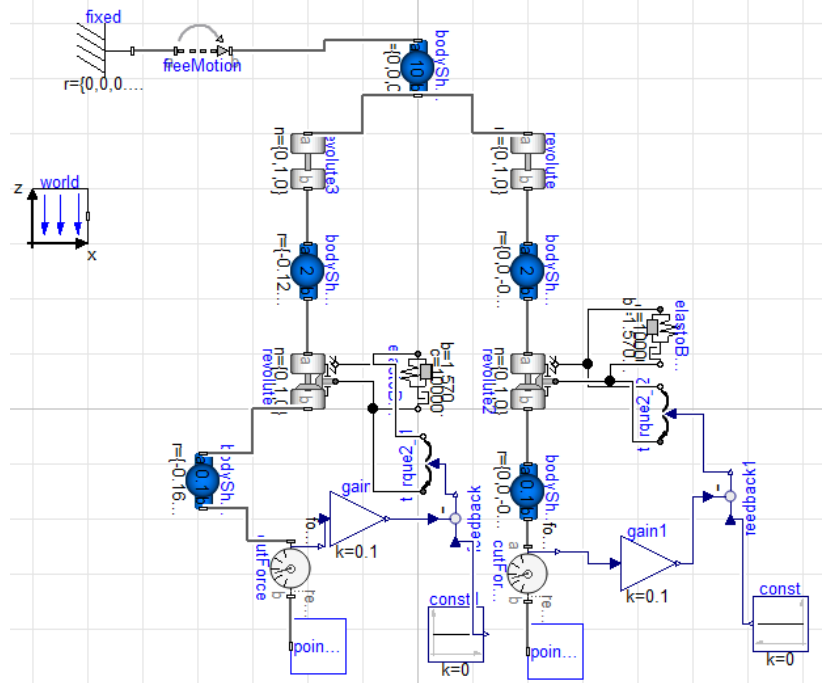

Figure 27 The passive dynamic walking model.

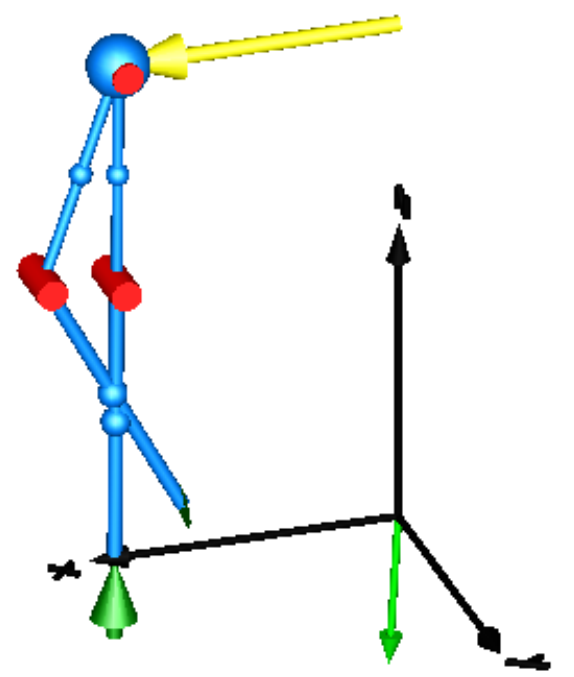

Figure 28 Animation view of the passive dynamic walking model.

In this model, 2 BodyShape components corresponding to the legs are connected to the PointContact component. The torque of the revolute joint corresponding to the knee is controlled as zero when the reaction force from the ground is zero. It holds the knee angle while the reaction force from the ground is above zero.

The gravity vector is tilted from $\mathrm{Z}$ axis. With the gravity the walking motion is continued. Since the legs of the model are placed at the same position in $\mathrm{Y}$ direction, the model fell down sideway after 10 steps.

The simulation can be animated with the skin of the human model installed with XVL Studio (Figure 29). 


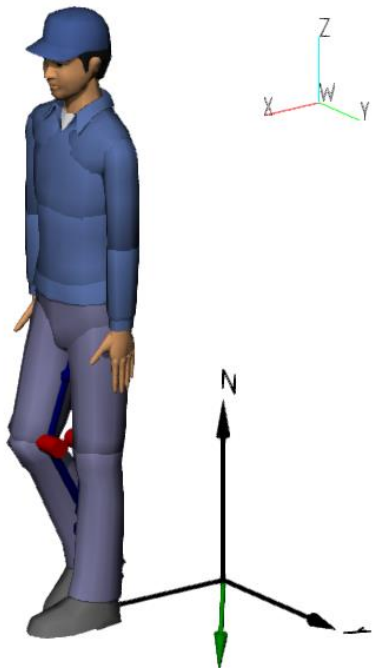

Figure 29 The walking model with the skin in XVL Studio.

\section{Future Work}

In the future work, we plan to extend the functionality of the prototype, and evaluate in the actual product design.

In the next prototype of the human model we will not use the muscle bone model. The forces of the muscle will be calculated from the torque of the joint inside the DMU Tool. Because of the detailed evaluation of each force of the muscles are not required in the use case of the DMU Tool. The reaction force on the ground described in the section 7.2 will be included in the next prototype. And the calculation of the gravity center of the whole body will be included. It helps to evaluate the working posture which is the main use case of the human model of the DMU Tool. In the Figure 30 left image is a typical human model holding a box which is created with XVL Studio. Since the gravity balance is not considered, the simulation model fall down foreword like the image right side.

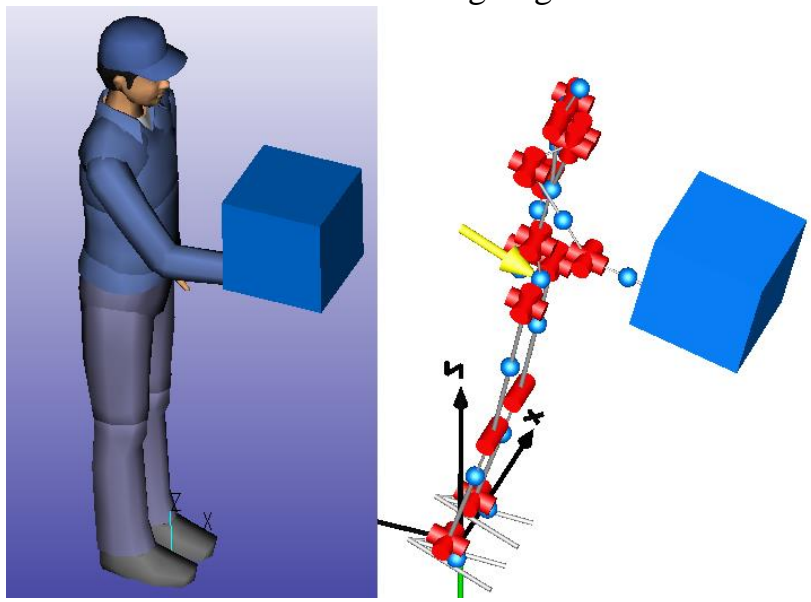

Figure 30 A typical human model holding a box.

Figure 31 shows the posture considered gravity balance. In this way, by using Modelica simulation in DMU Tool more natural posture can be created.

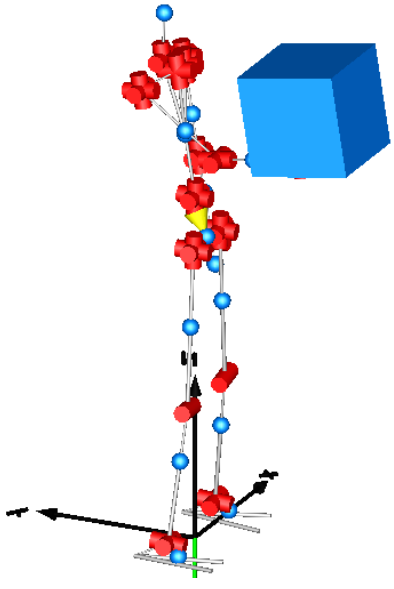

Figure 31

Also, the automatic generation of the models corresponding to human bodies of various physiques is planned.

\section{Conclusion}

This project demonstrated significant advantages using a DMU tool as a Modelica front-end. The advantages are as follows.

- Enables visualization of simulation results with 3D CAD models and/or 3D scan data.

- Enables easier 1D modeling by using the 3D user interface of the DMU Tool.

- Better visualization of results will promote the use of Model based design.

Further advantage can be expected with the future work described in the previous section.

\section{Acknowledgements}

The authors wish to thank Takayuki Kosaka (TADANO LTD.) and Marc Jablonski for their contributions and feedbacks. This research and the prototype were supported by New Energy and Industrial Technology Development Organization (NEDO) of Japan, and we would like to thank them for their assistance.

\section{References}

Autodesk (2016): BVH File Specification.

http://www.autodesk.com

Dassault Systèmes (2016): Dymola 2016

http://www.Dymola.com

David Mauricio Alba Lucero. (2012): Kinematic and Dynamic Analysis for Biped Robots Design.

Felix Oestersotebier, Peng Wang and Ansgar Trachtler. (2014): A Modelica Contact Library for Idealized Simulation of Independently Defined Contact Surfaces.

FMI-Standard.org (2014): Functional Mockup Interface for Model Exchange and Co-Simulation Version 2.0, July 25, 2014. https://www.fmi-standard.org 
Hilding Elmqvist, Sven Erik Mattsson, Christophe Chapuis. (2009): Redundancies in Multibody Systems and Automatic Coupling of CATIA and Modelica. In: Proceedings of the $7^{\text {th }}$ International Modelica Conference.

JModelica.org (2016): FMI Library 2.0.2

http://jmodelica.org

Lattice Technology (2016): XVL

http://www.lattice3d.com/

Martin Otter, Hilding Elmqvist and Sven Erik Mattsson

DLR; Dynasim: (2003): The New Modelica MultiBody Library. In: Proceedings of the $3^{\text {rd }}$ International Modelica Conference, Linkoping, November 3-4, 2003.

National Institute of Advanced Industrial Science and Technology (AIST) (2016): DhaibaWorks

http://www.dhaibaworks.com

Ohtomi, K. (2015): Kansei Modeling for Delight Design based on 1DCAE Concept. In: Proceedings of the $11^{\text {th }}$ International Modelica Conference, Versailles, France, September 21-23, 2015. doi: 10.3384/ecp15118

Toriya, H. (2008): 3D Manufacturing Innovation. doi: 10.1007/978-1-84800-038-4

Toriya, H. (2014): Manufacturing Innovation Based On Lightweight 3D Technology. In: The 4th IIEEJ International Workshop on Image Electronics and Visual Computing 2014.

Toriya H., Jablonski M. (2017): 3D Manufacturing Evolution: Evolutionary Change in Global Manufacturing with Digital Data. ASIN: B01N29ZFZM 